\title{
Left Atrial Tumor Thrombus in a Patient With Squamous Cell Carcinoma of Lung
}

\author{
Ghulam Murtaza ${ }^{\mathrm{a}, \mathrm{c}}$, Muhammad Khalid ${ }^{\mathrm{a}}$, Abdul Khan ${ }^{\mathrm{a}}$, \\ Lance Klosterman ${ }^{\mathrm{b}}$, Terry Forrest ${ }^{\mathrm{b}}$
}

\begin{abstract}
Tumor thrombus in left atrium (LA) is very rare but a serious complication in patients with malignancy. It has a significant impact on clinical decision making, staging and prognosis in oncological patients. Tumor thrombus is seen in a wide variety of malignancies. While rare, lung cancer tumor thrombus may occur. Rarely, it can even extend into LA. Usually, it is managed by surgical resection with chemotherapy. We describe a rare case of tumor thrombus in a patient with squamous cell carcinoma of lung that was managed with chemoand radiation therapy.
\end{abstract}

Keywords: Atrium; Tumor; Thrombus; Cancer; Surgery

\section{Introduction}

Tumor thrombus extension into left atrium (LA) from lung cancer is seen infrequently. There are no standardized management approaches for this complication. Usually treatment is surgical resection for intra-atrial extension of tumor thrombus. We present a case of lung cancer in a patient who was found to have superior vena cava (SVC) obstruction and had LA tumor thrombus managed without surgical resection.

\section{Case Report}

An 80-year-old man with history of grade 1 follicular lymphoma since 2013 with no indication for therapy being followed by oncology for clinical surveillance presented with worsening shortness of breath and hemoptysis. Physical exam

Manuscript submitted September 3, 2019, accepted September 9, 2019

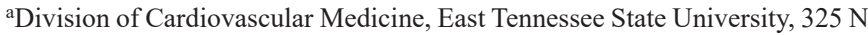
state of Franklin Road, Johnson City, TN 37604, USA

bivision of Cardiovascular Medicine, Mountain Home VA Medical Center, Lamont Street and Veterans Way, Johnson City, TN 37604, USA

${ }^{\mathrm{c} C}$ Corresponding Author: Ghulam Murtaza, Division of Cardiovascular Medicine, East Tennessee State University, 325 N state of Franklin Road, Johnson City, TN 37604, USA. Email: murtazag@etsu.edu

doi: https://doi.org/10.14740/cr943 was unremarkable, and lab workup showed chronic anemia. Computerized tomography (CT) imaging of the chest showed an enlarging $2.0 \mathrm{~cm}$ density in the posterior right upper lobe of lung (RUL) as well as scattered pulmonary nodules in the RUL. With his extensive history of smoking, he underwent bronchoscopy with endobronchial ultrasound-guided biopsy (EBUS) of mediastinal lymphadenopathy. Bronchoscopy revealed lung mass with endobronchial lesions and mediastinal lymphadenopathy. Although clinically on bronchoscopy it appeared to be cancer, biopsy was negative. He subsequently was sent to interventional radiology for biopsy of an RUL spiculated mass, which again was negative for cancer. Needle biopsy sections of lungs were suggestive of organizing pneumonia.

With clinically suspected cancer and two separate negative biopsies, we decided to proceed with positron emission tomography (PET) scan to reassess approaches for tissue biopsy. Meanwhile patient was started on antibiotics with which he had significant improvement clinically and his shortness of breath and mucus production got better. Patient had PET scan that showed extensive mediastinal lymphadenopathy throughout the right mediastinum and LA which could probably correspond to patient's known lymphoma (Figs. 1 and 2) as his biopsied right spiculated lung mass did not appear to be growing and had less impressive uptake. Patient was recommended to have repeat EBUS; however, he refused to undergo any further procedures or biopsies.

Three months later, patient underwent repeat chest CT with contrast that showed right hilar and mediastinal mass measuring $7 \mathrm{~cm}$ in size with involvement of right and left brachiocephalic veins, SVC and a large tumor thrombus in the LA (Fig. 3). He had transthoracic echocardiogram done that showed large left atrial mass $3.8 \times 3.2 \mathrm{~cm}$ lying in the base of the LA extending into the main body of the LA (Figs. 4 and 5). Patient eventually decided to have repeat bronchoscopy with EBUS to biopsy the mediastinal mass before chemotherapy could be considered. He was also started on radiation therapy after biopsy performed with his SVC syndrome. Because of SVC narrowing and possible complete obstruction with collateralization, he also underwent SVC stent placement as well (Fig. 6). His biopsy results came back showing squamous cell carcinoma (SCC) of lung. Patient then underwent combined chemotherapy with platinum-based agents for lung cancer and radiation therapy. Patient had repeat echocardiogram done 6 weeks later that showed the large mass in the LA was no longer visualized (Fig. 7). On his repeat chest $\mathrm{CT}$, there was significant decrease in right mediastinal mass and left atrial mass (Fig. 8). Patient underwent 


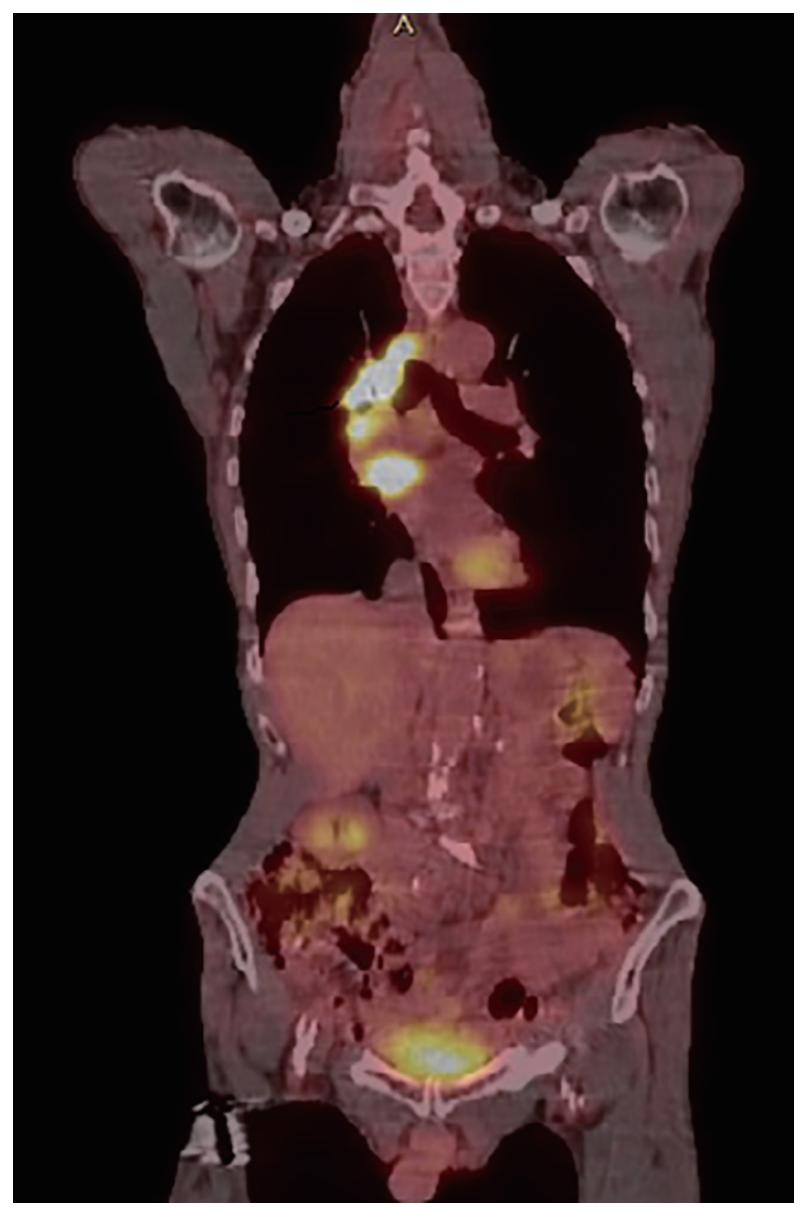

Figure 1. PET/CT longitudinal view showing abnormal uptake throughout right mediastinum and LA. PET/CT: positron emission tomography/ computerized tomography; LA: left atrium.

systemic chemotherapy, improved clinically over long-term follow-up and is under oncological surveillance.

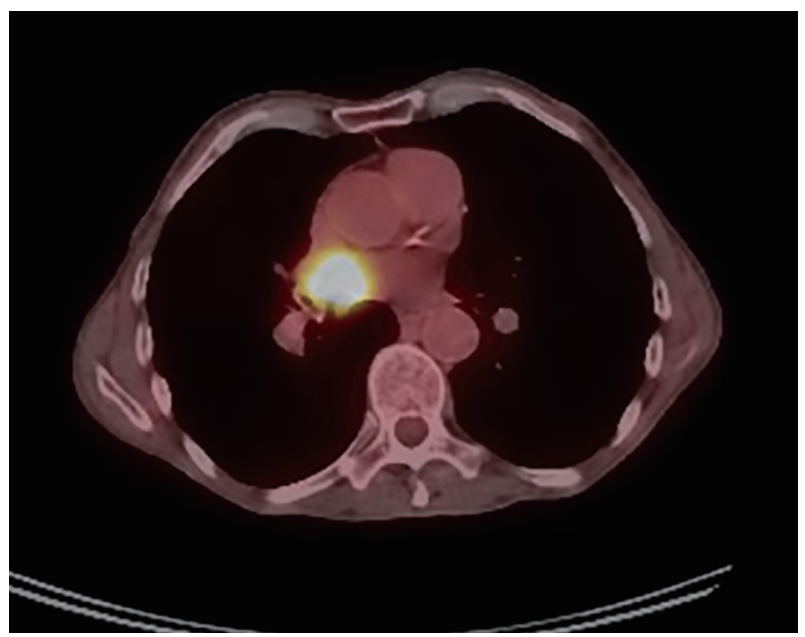

Figure 2. PET/CT cross-sectional view showing abnormal uptake throughout the right mediastinum and the LA. PET/CT: positron emission tomography/computerized tomography; LA: left atrium.
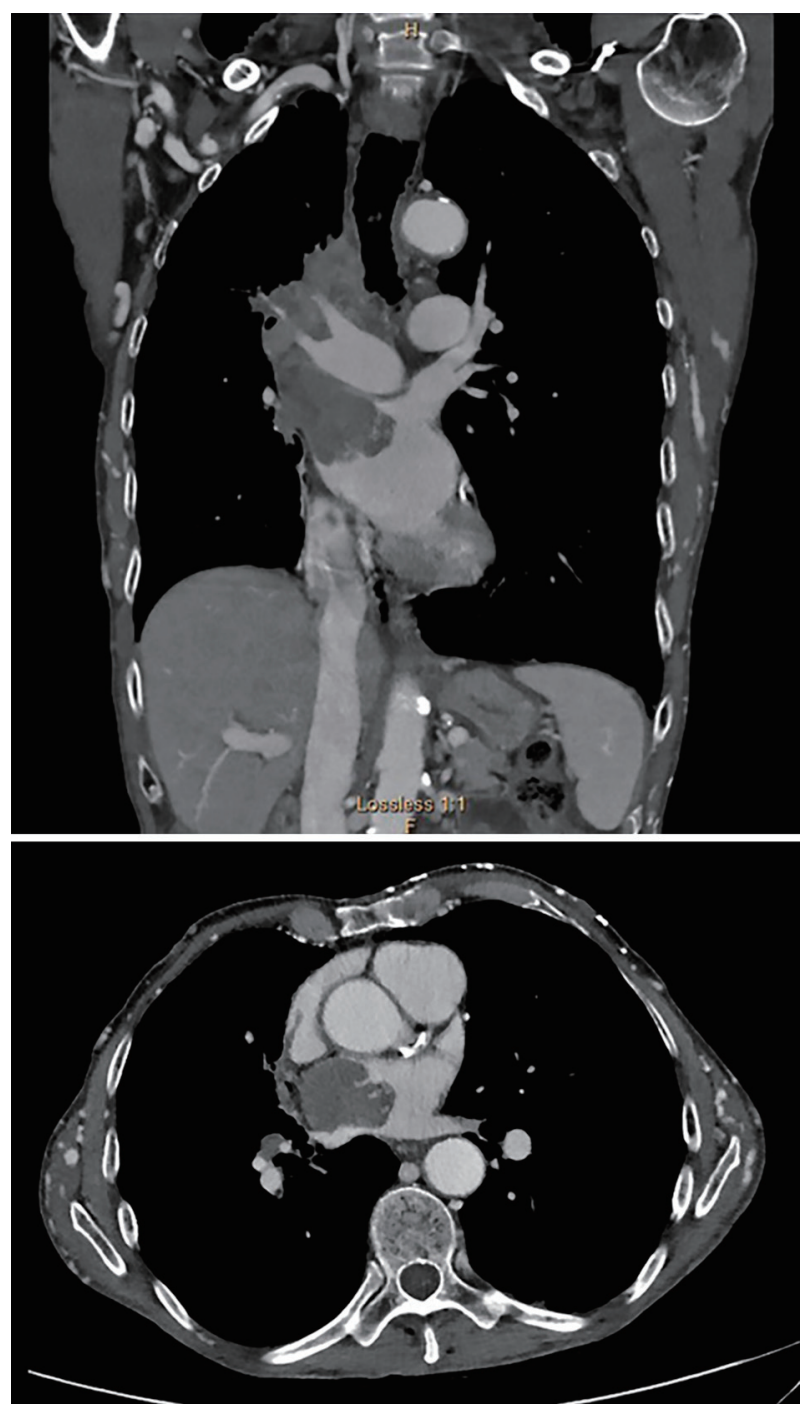

Figure 3. Right hilar mass eroding into the LA. Notice multiple chest wall collaterals secondary to SVC occlusion. LA: left atrium; SVC: superior vena cava.

\section{Discussion}

Intra-cardiac tumor extension also known as tumor thrombus can be seen in many different types of cancer [1]. This is most frequently seen in hepatocellular carcinoma, renal cell carcinoma (RCC) and adrenal cortical carcinoma (ACC). In patients with RCC, tumor thrombus extends along the inferior vena cava as a result of underlying $\mathrm{RCC}$ or along the portal vein from hepatocellular carcinoma (HCC) [2]. Lung cancer can invade SVC causing SVC syndrome [3]. However, it is very rare for patients with non-small lung cancer to have extensive SVC thrombosis with erosion into LA causing almost complete obliteration of left atrial cavity [4].

Tumor thrombus is generally found incidentally during staging of the disease. In the past venography showing filling defects within the affected vessel was performed to aid the diagnosis. However, these days cardiac CT/magnetic resonance 


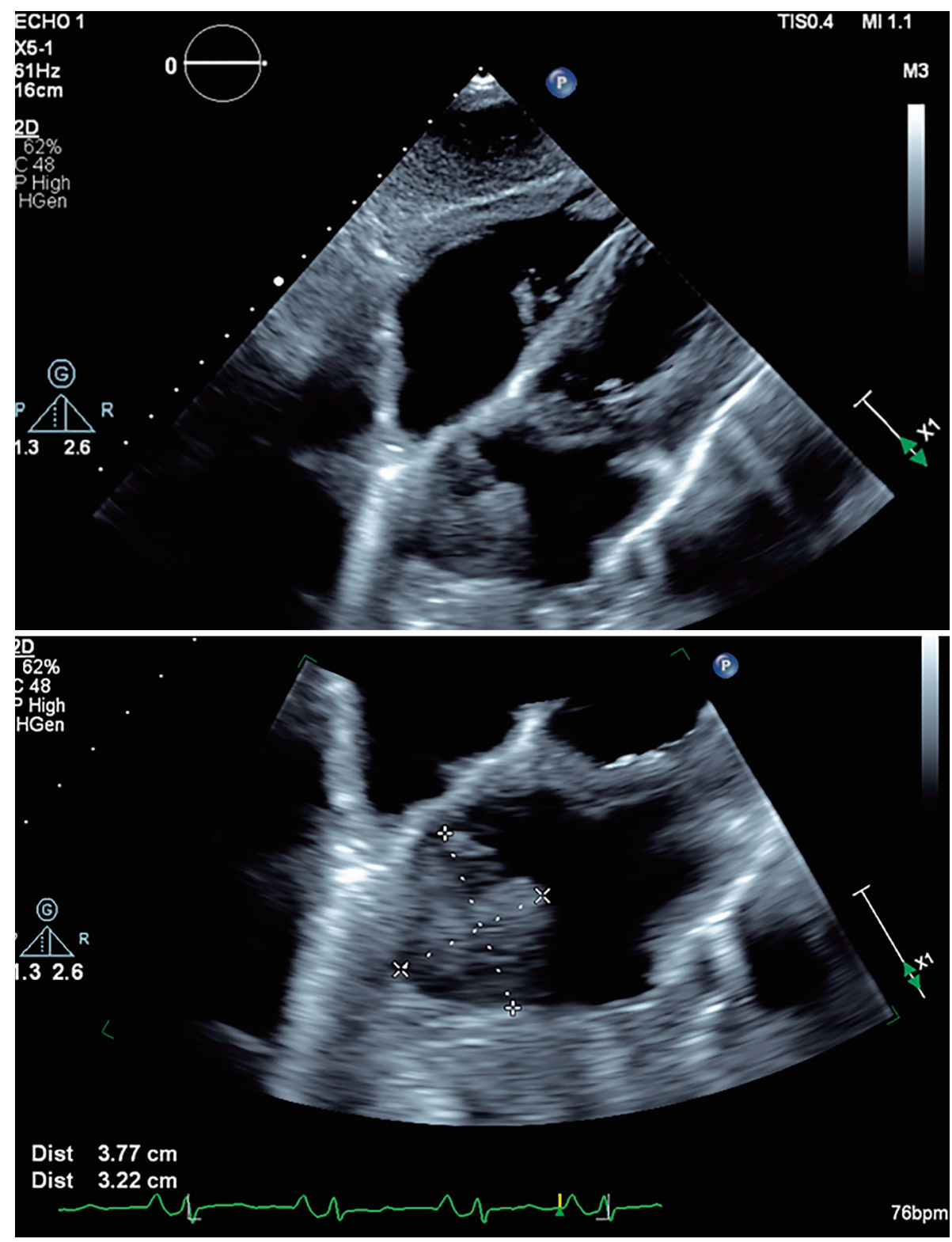

Figure 4. Subcostal views showing large left atrial mass measured $3.8 \times 3.2 \mathrm{~cm}$ in left atrial cavity.

(MR) techniques are commonly used [5]. With cardiac CT/ $\mathrm{MR}$, one can not only detect tumor thrombus but also visualize its extension and distinguish it from bland thrombus. Following that echocardiography is the imaging technique used to further delineate characteristics of a mass such as its mobility, attachment and potential hemodynamic consequences. It also allows for serial imaging over time without the need for radiation, iodine or gadolinium contrast agents. In addition to cardiac CT/MR, echo contrast agents are also useful to characterize the intra-cardiac mass further by evaluating the extent of contrast enhancement. Malignant tumors demonstrate hyperenhancement with contrast, whereas thrombi do not appear to enhance [6].

The presence of tumor thrombus is associated with poor prognosis. Patients with lung cancer with tumor thrombus should undergo urgent evaluation and treatment. Majority of these patients undergo complete surgical resection of the primary tumor and tumor thrombus with cardiopulmonary bypass [7]. However, there are no standard surgical feasibility criteria established for resection of LA tumor thrombus. Also resection of the LA because of tumor invasion is an infrequent procedure, and rarely performed especially when there is extensive left atrial involvement. Use of neo-adjuvant chemotherapy and radiation therapy depends on underlying malignancy. Use of prophylactic anticoagulation can be considered as well [8].

In our case, the differential diagnosis of left atrial tumor thrombus included from possible lymphoma vs. SCC. His hilar mass eroding into LA was biopsy proven to be SCC. With his advanced age and comorbidities, we decided to proceed with 


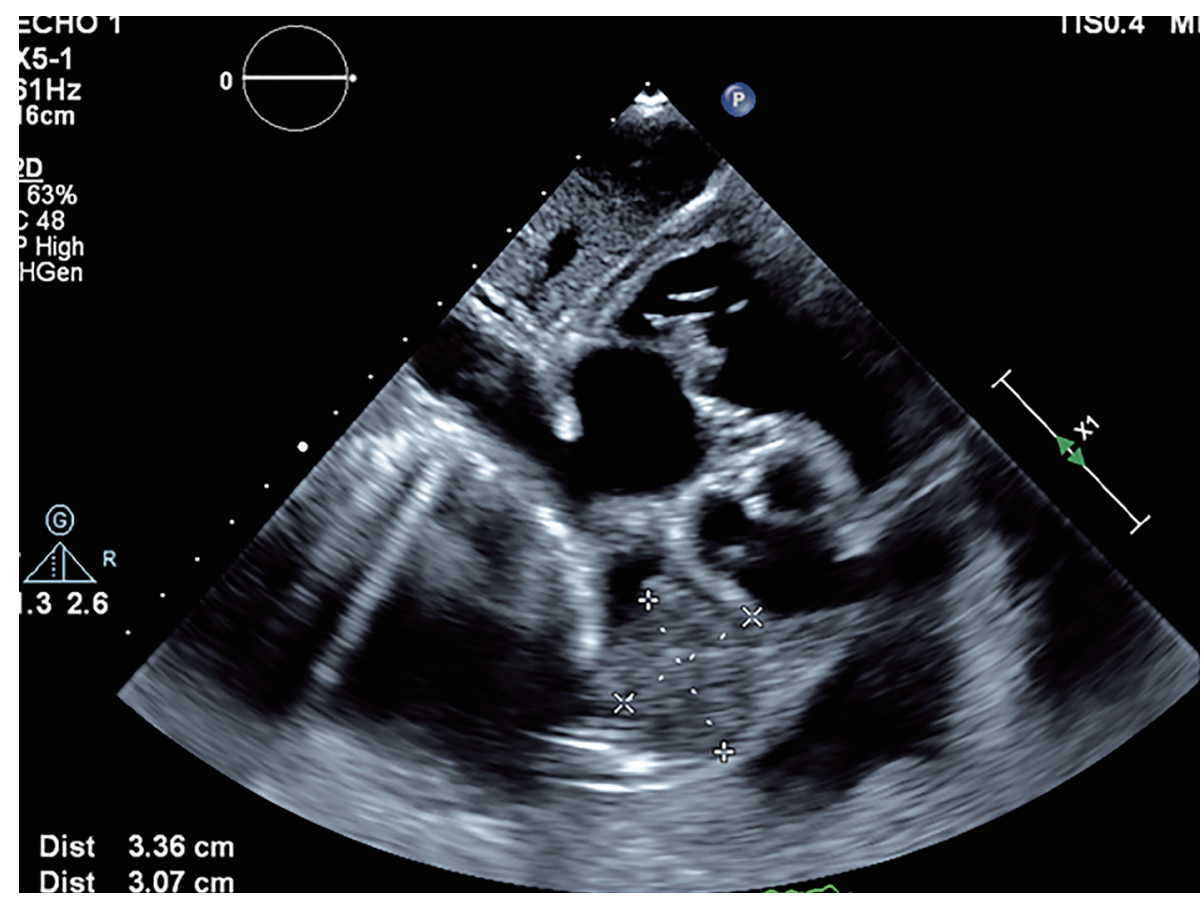

Figure 5. Subcostal five-chamber view showing almost complete obliteration of left atrial cavity.

chemotherapy and radiotherapy. Also with his extensive supraventricular tachycardia syndrome, he underwent SVC stent placement. With his treatment, there was significant reduction in size of hilar mass and resolution of left atrial tumor thrombus on serial imaging with echo and CT. To our knowledge, there are no previous cases of SCC with tumor thrombus in the LA reported in literature.

\section{Conclusion}

Lung cancer with tumor thrombus is a serious complication

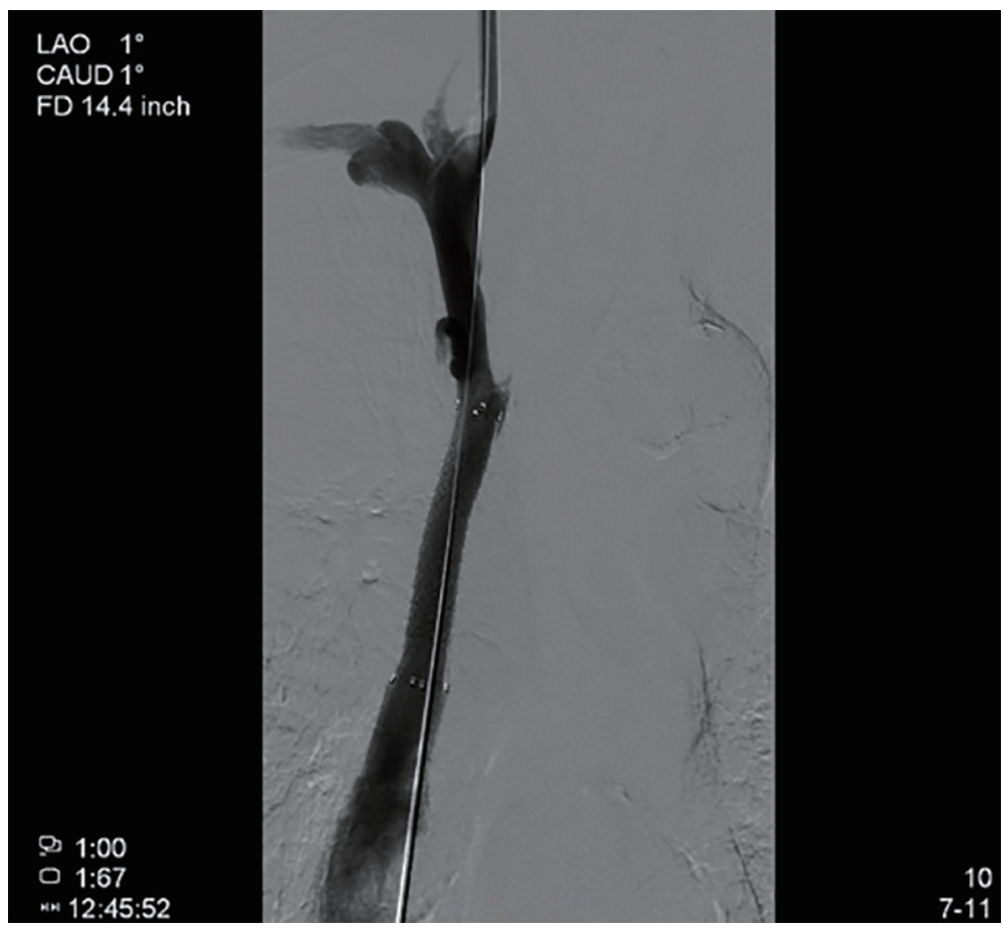

Figure 6. Angiographic image of SVC after stenting with $10 \times 60 \mathrm{~mm}$ Fluency stent. SVC: superior vena cava. 


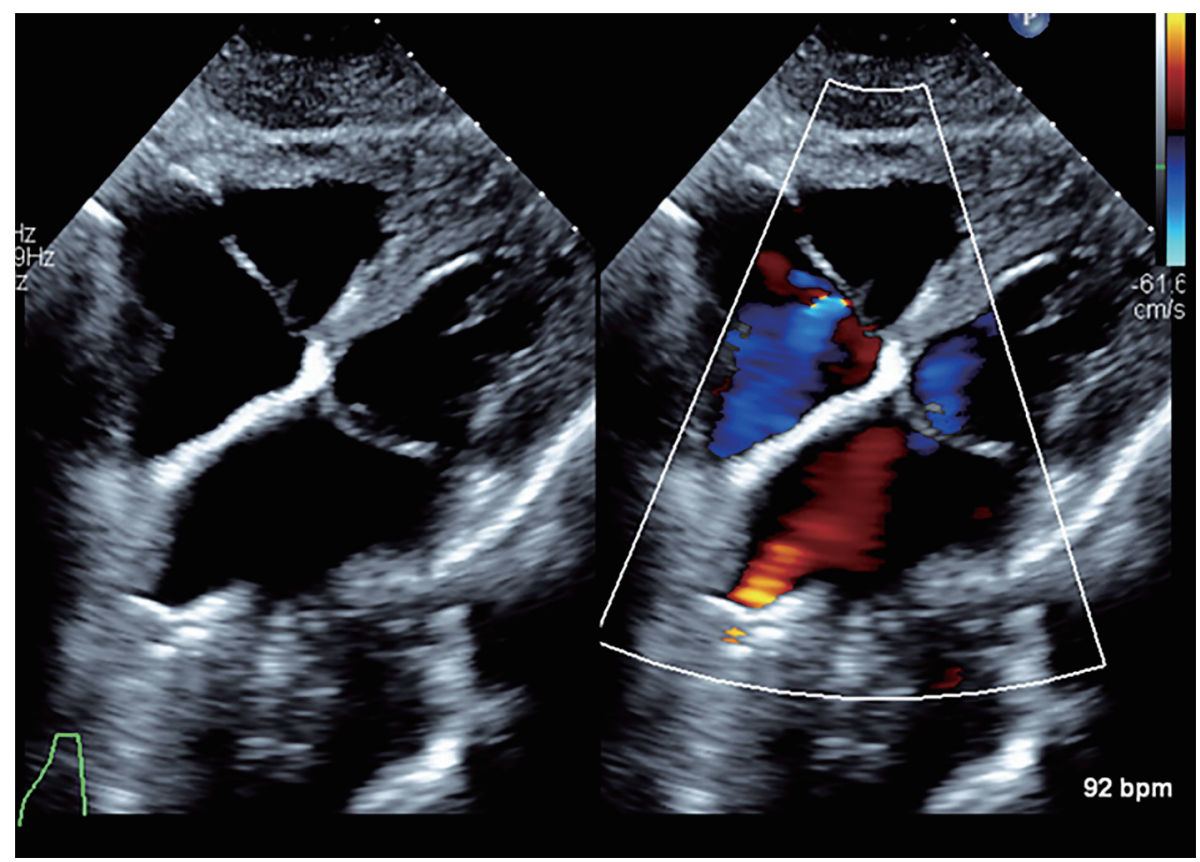

Figure 7. Resolution of left atrial tumor thrombus on echo 6 weeks after chemo- and radiotherapy.

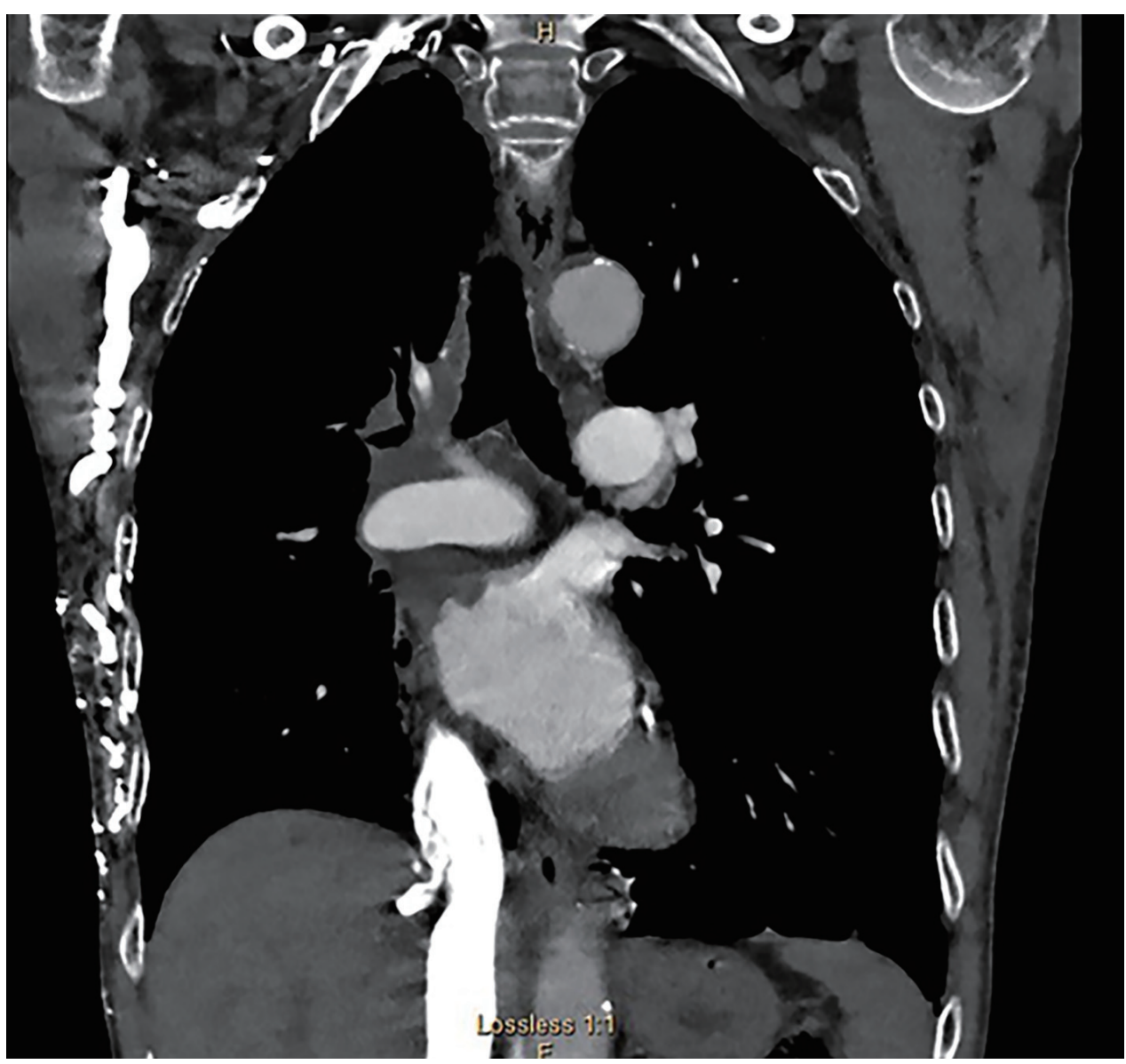

Figure 8. Decrease in size of right mediastinal and left atrial mass. Notice multiple chest wall collaterals secondary to SVC occlusion. SVC: superior vena cava. 
especially with involvement of LA and concomitant SVC syndrome. Multidisciplinary approach is needed in management of these patients. Surgical intervention with extensive involvement of LA can be challenging. Further studies are needed to evaluate role of conservative management in selective cases who are not surgical candidates.

\section{Acknowledgments}

None to declare.

\section{Financial Disclosure}

None to declare.

\section{Conflict of Interest}

None to declare.

\section{Informed Consent}

Obtained for this publication.

\section{Author Contributions}

GM and MK wrote the paper. LK and AK performed collection of images. TF reviewed paper and helped with final review of the article.

\section{References}

1. Quencer KB, Friedman T, Sheth R, Oklu R. Tumor thrombus: incidence, imaging, prognosis and treatment. Cardiovasc Diagn Ther. 2017;7(Suppl 3):S165-S177.

2. Zisman A, Wieder JA, Pantuck AJ, Chao DH, Dorey F, Said JW, Gitlitz BJ, et al. Renal cell carcinoma with tumor thrombus extension: biology, role of nephrectomy and response to immunotherapy. J Urol. 2003;169(3):909916.

3. Lee D, Moon SM, Kim D, Kim J, Chang H, Yang B, Jeong $\mathrm{SH}$, et al. Lung cancer with superior vena cava syndrome diagnosed by intravascular biopsy using EBUS-TBNA. Respir Med Case Rep. 2016;19:177-180.

4. Xie D, Ding J, Zhou X, Chen C, Zhao D, Jiang G. Tumor thrombus in left atrium from pulmonary adenosquamous carcinoma. Asian Cardiovasc Thorac Ann. 2015;23(1):7577.

5. Kassop D, Donovan MS, Cheezum MK, Nguyen BT, Gambill NB, Blankstein R, Villines TC. Cardiac masses on cardiac CT: a review. Curr Cardiovasc Imaging Rep. 2014;7:9281.

6. Mankad R, Herrmann J. Cardiac tumors: echo assessment. Echo Res Pract. 2016;3(4):R65-R77.

7. Wiebe K, Baraki H, Macchiarini P, Haverich A. Extended pulmonary resections of advanced thoracic malignancies with support of cardiopulmonary bypass. Eur J Cardiothorac Surg. 2006;29(4):571-577; discussion 577-578.

8. Woodruff DY, Van Veldhuizen P, Muehlebach G, Johnson P, Williamson T, Holzbeierlein JM. The perioperative management of an inferior vena caval tumor thrombus in patients with renal cell carcinoma. Urol Oncol. 2013;31(5):517-521. 